\title{
A putative miRNA in the spike gene of SARS-CoV-2 has perfect sequence identity to both the forward and reverse complementary strands of hsa-mir-8055 involved in T- cell response to antigen
}

\section{Resume}

The Severe Acute Respiratory Syndrome Coronavirus 2 (SARS-CoV-2), also known as COVID-19, encodes for a spike protein that is responsible for both attachment and membrane fusion, thereby being critical in the pathogenicity of this virus. Here, I report that a putative miRNA localized in the spike gene of SARS-CoV-2 matches to the forward strand of hsa-miR8055, a miRNA expressed during T-cell response to antigen, and also binds with perfect complementarity to its seed region.

\section{Main Text}

The Severe Acute Respiratory Syndrome Coronavirus 2 (SARS-CoV-2) spike protein is responsible for both virus attachment and fusion, being therefore critical in the pathogenicity of SARS-CoV-2. Two structural features of this spike protein have been proposed to increase the pathogenicity of SARS-CoV-2: 1-an insertion in the open reading frame of 12 nucleotides, with generation of a functional furin-cleavage motif (RRAR), and 2-three O-glycan residues linked to this site (Andersen et al. 2020).

Small interfering RNAs (siRNAs) and microRNAs (miRNAs) are regulatory small non coding RNA (sncRNA) molecules that suppress gene expression by binding to the $3^{\prime}$ region of target mRNAs. sncRNA-based strategies have already been employed to inhibit the expression of the SARS-CoV spike gene (Qin 2004). The prediction of sncRNAs encoded from the SARS-CoV2 genome or with similarity to the SARS-CoV spike-coding sequence may have implications in designing sncRNA-based therapies for effective antiviral silencing. Importantly for in silico analyses, evidence supports the fact that molecular mechanisms of host miRNAs against viruses are sequence-specific rather than species-specific. Plant viruses have shown sequence similarity to human miRNAs and even exhibit perfect matches with their seed sequences (RebolledoMendez et al. 2013). Similarly, bioinformatics approaches have recently predicted crosskingdom interactions between plant miRNAs and human viruses (Barozai and Din 2017).

Here I report that pab-miR11409d, a miRNA expressed in the gymnosperm Picea abies L. (Xia et al. 2015), exhibits nucleotide identity to the $3^{\prime}$ coding region of the SARS-CoV-2 spike gene (NCBI Accession number LC528233.1). The importance of this exploratory analysis is twofold: first, the identification of miRNA-like elements in SARS-CoV-2; and second, the fact that mature miRNAs can be inhibited with antisense oligonucleotides (antagomirs). It is also 
important to note that plant microRNAs have shown potential to cross kingdom barriers for regulating target gene expression in mammalian cells (Zhang et al. 2012, Liang et al 2015, Chin et al. 2016). Therefore, in the hypothetical case that pab-miR11409d evolved as a defense mechanism associated with viral infections, its utilization could be helpful for the treatment of SARS-CoV-2. Moreover, in this study, it has been found that the putative miRNA matches to hsa-miR-8055, a miRNA expressed during T-cell response to antigen (Zhang et al. 2019). Interestingly, Zhang et al. (2019) proposed that a circular RNA (circ 0071542) has a proinflammatory role in fulminant myocarditis by sponging miR-8055. Even more interesting is the fact that this viral sequence also binds with perfect complementarity to the seed region of hsa-miR-8055 (see below In silico analyses). It seems that this viral mechanism may be evolutionarily conserved in nature. Recently, Gorbea et al. (2019) reported two viral sncRNAs encoded by a herpesvirus that bind with perfect complementarity to the seed regions of miR$142-3 p$ and miR-16 in order to promote the destabilization of target mRNAs, and thus downregulate the expression of apoptotic proteins in infected T cells.

\section{References}

Andersen, K. G., Rambaut, A., Lipkin, W. I., Holmes, E. C., \& Garry, R. F. (2020). The proximal origin of SARS-CoV-2. Nature Medicine, 1-3.

Barozai, M. Y. K., \& Din, M. (2017, January). Initial screening of plant most conserved MicroRNAs targeting infectious viruses: HBV and HCV. In 2017 14th International Bhurban Conference on Applied Sciences and Technology (IBCAST) (pp. 192-196). IEEE.

Chin, A. R., Fong, M. Y., Somlo, G., Wu, J., Swiderski, P., Wu, X., \& Wang, S. E. (2016). Cross-kingdom inhibition of breast cancer growth by plant miR159. Cell research, 26(2), 217228.

Gorbea, C., Mosbruger, T., Nix, D. A., \& Cazalla, D. (2019). Viral miRNA adaptor differentially recruits miRNAs to target mRNAs through alternative base-pairing. eLife, 8 .

Griffiths-Jones, S., Saini, H. K., van Dongen, S., \& Enright, A. J. (2007). miRBase: tools for microRNA genomics. Nucleic acids research, 36(suppl_1), D154-D158.

Krüger, J., \& Rehmsmeier, M. (2006). RNAhybrid: microRNA target prediction easy, fast and flexible. Nucleic acids research, 34(suppl_2), W451-W454.

Liang, H., Zhang, S., Fu, Z., Wang, Y., Wang, N., Liu, Y., ... \& Chen, X. (2015). Effective detection and quantification of dietetically absorbed plant microRNAs in human plasma. The Journal of nutritional biochemistry, 26(5), 505-512.

Qin, Z. L., Zhao, P., Zhang, X. L., Yu, J. G., Cao, M. M., Zhao, L. J., ... \& Qi, Z. T. (2004). Silencing of SARS-CoV spike gene by small interfering RNA in HEK 293T cells. Biochemical and biophysical research communications, 324(4), 1186-1193.

Qureshi, A., Thakur, N., Monga, I., Thakur, A., \& Kumar, M. (2014). VIRmiRNA: a comprehensive resource for experimentally validated viral miRNAs and their targets. Database, 2014. 
Rebolledo-Mendez, J., Vaishnav, R., Cooper, N., \& Friedland, R. (2013). Cross-kingdom sequence similarities between human micro-RNAs and plant viruses. Communicative \& integrative biology, 6(5), e24951.

Xia, R., Xu, J., Arikit, S., \& Meyers, B. C. (2015). Extensive families of miRNAs and PHAS loci in Norway spruce demonstrate the origins of complex phasiRNA networks in seed plants. Molecular biology and evolution, 32(11), 2905-2918.

Zhang, L., Hou, D., Chen, X., Li, D., Zhu, L., Zhang, Y., ... \& Yin, Y. (2012). Exogenous plant MIR168a specifically targets mammalian LDLRAP1: evidence of cross-kingdom regulation by microRNA. Cell research, 22(1), 107-126.

Zhang, L., Han, B., Wang, J., Liu, Q., Kong, Y., Jiang, D., \& Jia, H. (2019). Differential expression profiles and functional analysis of circular RNAs in children with fulminant myocarditis. Epigenomics, 11(10), 1129-1141. 


\section{In silico analyses}

\section{Identification of a putative SARS-CoV-2 encoded-miRNA}

The last portion (1000 nucleotides) in the 3' coding region of the SARS-CoV-2 spike gene was mapped to identify sequences with similarity to known mature miRNAs in miRbase (Griffiths-Jones et al. 2007).

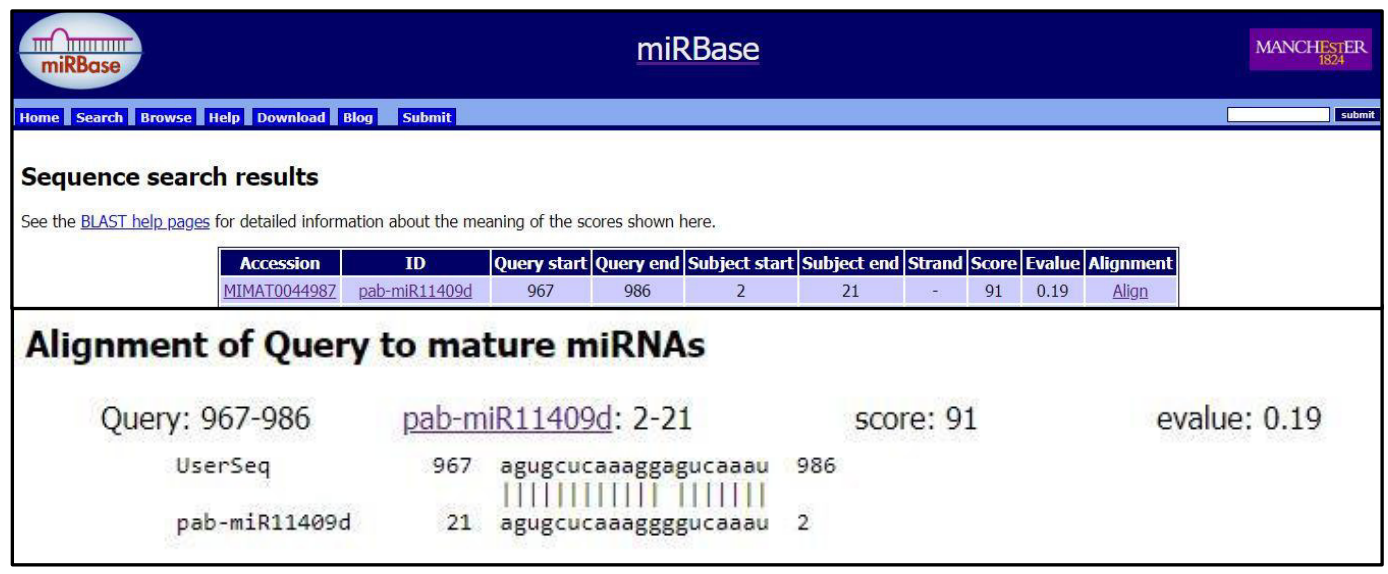

The aligned sequence in the $3^{\prime}$ region of the SARS-CoV-2 spike gene is shown in blue color. Letters below nucleotide sequence indicate the amino acid sequence in the single letter code.

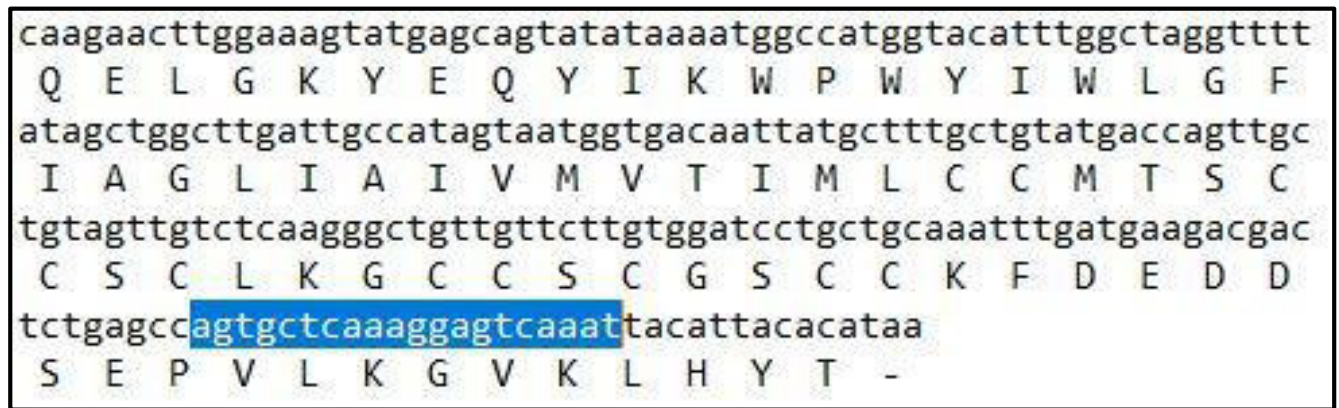

$>$ putative SARS-CoV-2 encoded-miRNA

AGTGCTCAAAGGAGTCAAAT 
The VIRmiRNA (Qureshi et al. 2014) bioinformatic tool was used to identify matches of the putative SARS-CoV-2 miRNA against human miRNAs, and nucleotide sequence identity with hsamir-8055 was observed as shown in the bottom Figure:

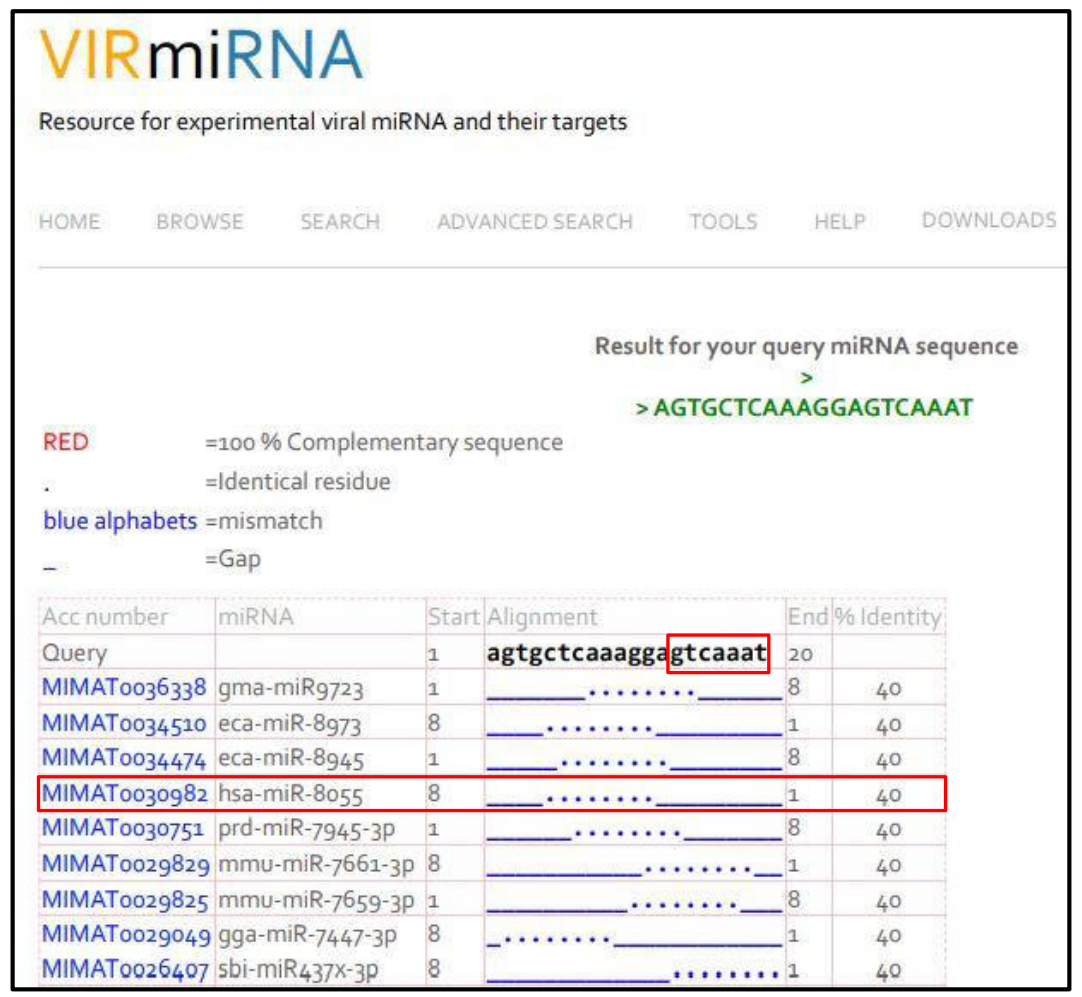

>hsa-mir-8055 MI0025891 (forward stem-loop sequence)

TTACGGTCACACTGTGCTCACTTTGTCAAATAAAAGATGGAGTCGTTTGACGTCTTTTAACACCCTTTGA GCACATGAGCAGACGGAGTGATCATGA

Interestingly, the putative viral miRNA also displays perfect complementarity to the seed region of hsa-miR-8055:

$>$ putative SARS-CoV-2 encoded-miRNA

AGTGCTCAAAGGAGTCAAAT

>hsa-mir-8055 MI0025891 (reverse complementary strand)

TCATGATCACTCCGTCTGCTCATGTGCTCAAAGGGTGTTAAAAGACGTCAAACGACTCCATCTTTTATTT GACAAAGTGAGCACAGTGTGACCGTAA 
As is displayed in the Figure, the putative miRNA localized in the SARS-CoV-2 spike gene also binds with perfect complementarity to the seed region of hsa-mir-8055 (CGAGUUUC, letters in pink color). The alignments between the SARS-CoV-2 spike gene and both forward and reverse complementary strands of hsa-mir-8055 are represented by top and bottom black boxes, respectively.

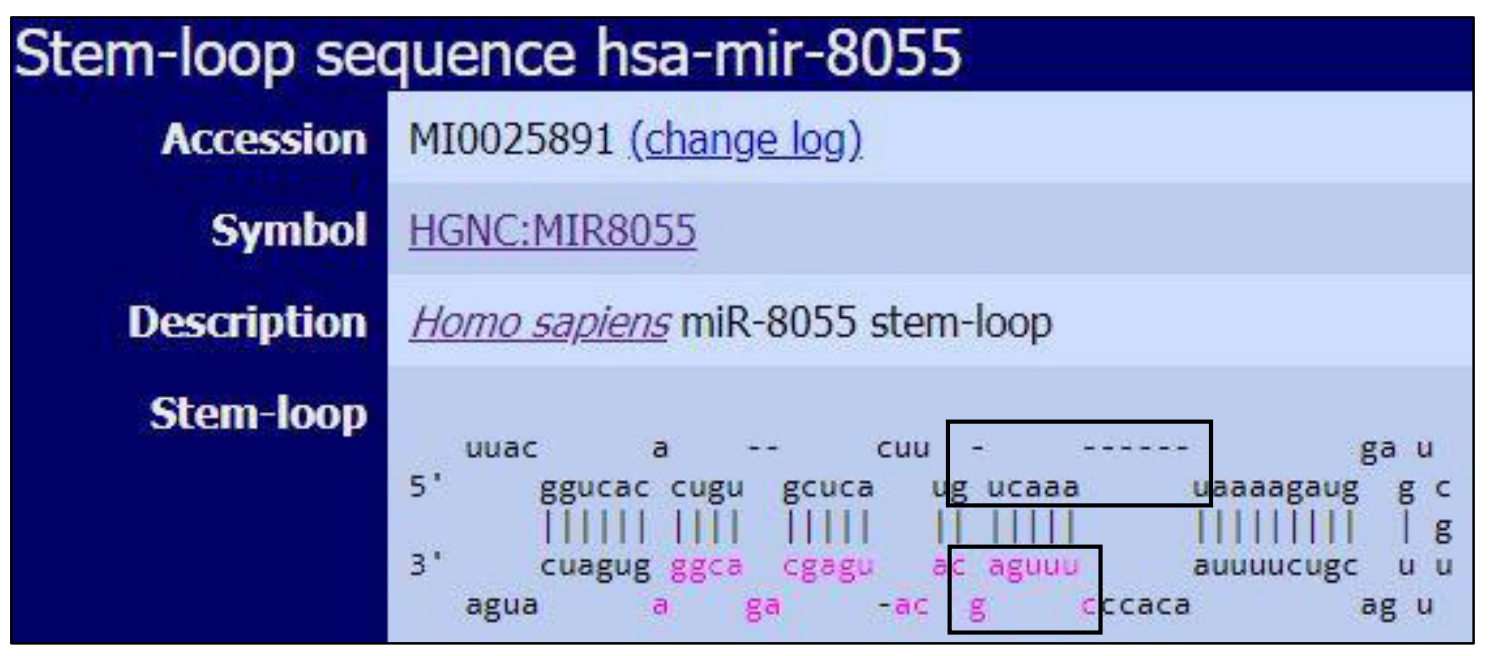


Version: RNAhybrid 2.2

searching

dataset: 1

mde of SARS-CoV-2_seq: -39.599998

Individual hits

dataset: 1

Target: pab-MIR11409d

length: 98

MiRNA: SARS-CoV-2_seq

length: 20

mfe: $-33.2 \mathrm{kcal} / \mathrm{mol}$

p-value: undefined

Position: 71

target 5' C

AUUUGAC CCUUUGAGCACU

UAAACUG GGAAACUCGUGA

miRNA $3^{\prime}$

A

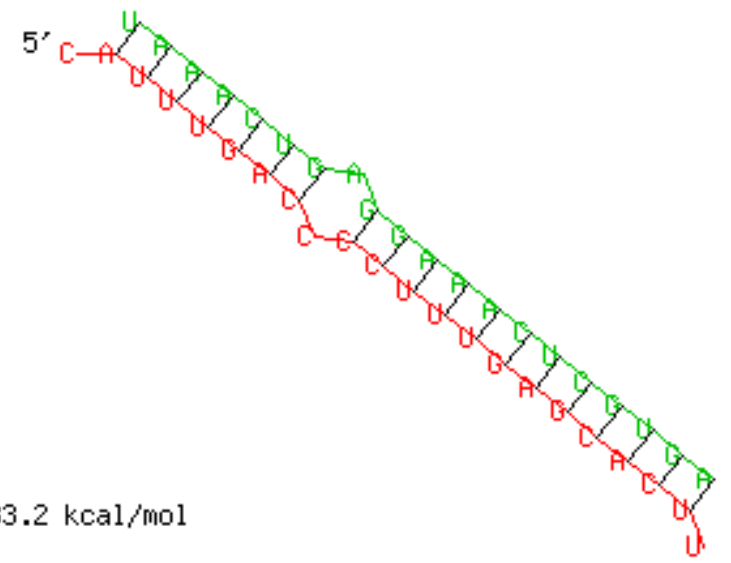

plot as png,jpeg or ps (in a new window) 
Version: RNAhybrid 2.2

searching

dataset: 1

mde of SARS-CoV-2_seq: -39.599998

Individual hits

dataset: 1

Target: hsa-mir-8055

length: 97

MiRNA: SARS-CoV-2_seq

length: 20

mfe: $-28.9 \mathrm{kcal} / \mathrm{mol}$

p-value: undefined

Position: 44

target $5^{\prime} \mathrm{C}$

GUCUUUUAACAC

GUUUGAC

UAAACUG

$A$

CCUUUGAGCAC

miRNA $3^{\prime}$

GGAAACUCGUG

plot as png,jpeg. or ps (in a new window)

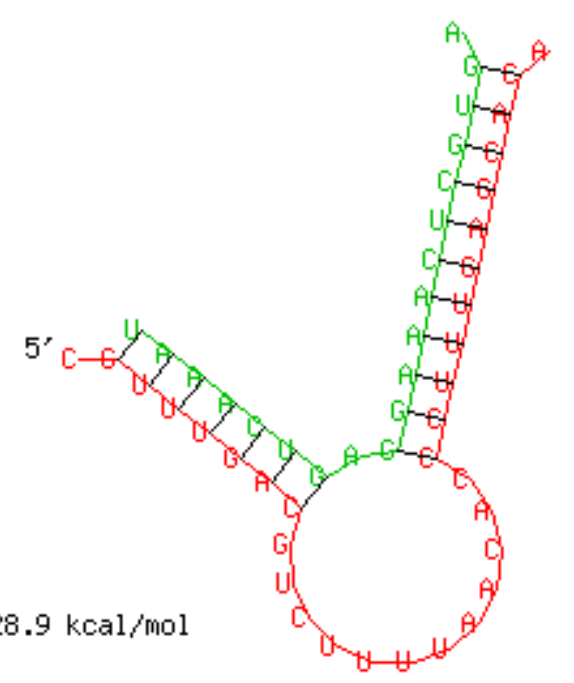




\section{Stem-loop sequence hsa-mir-8055}

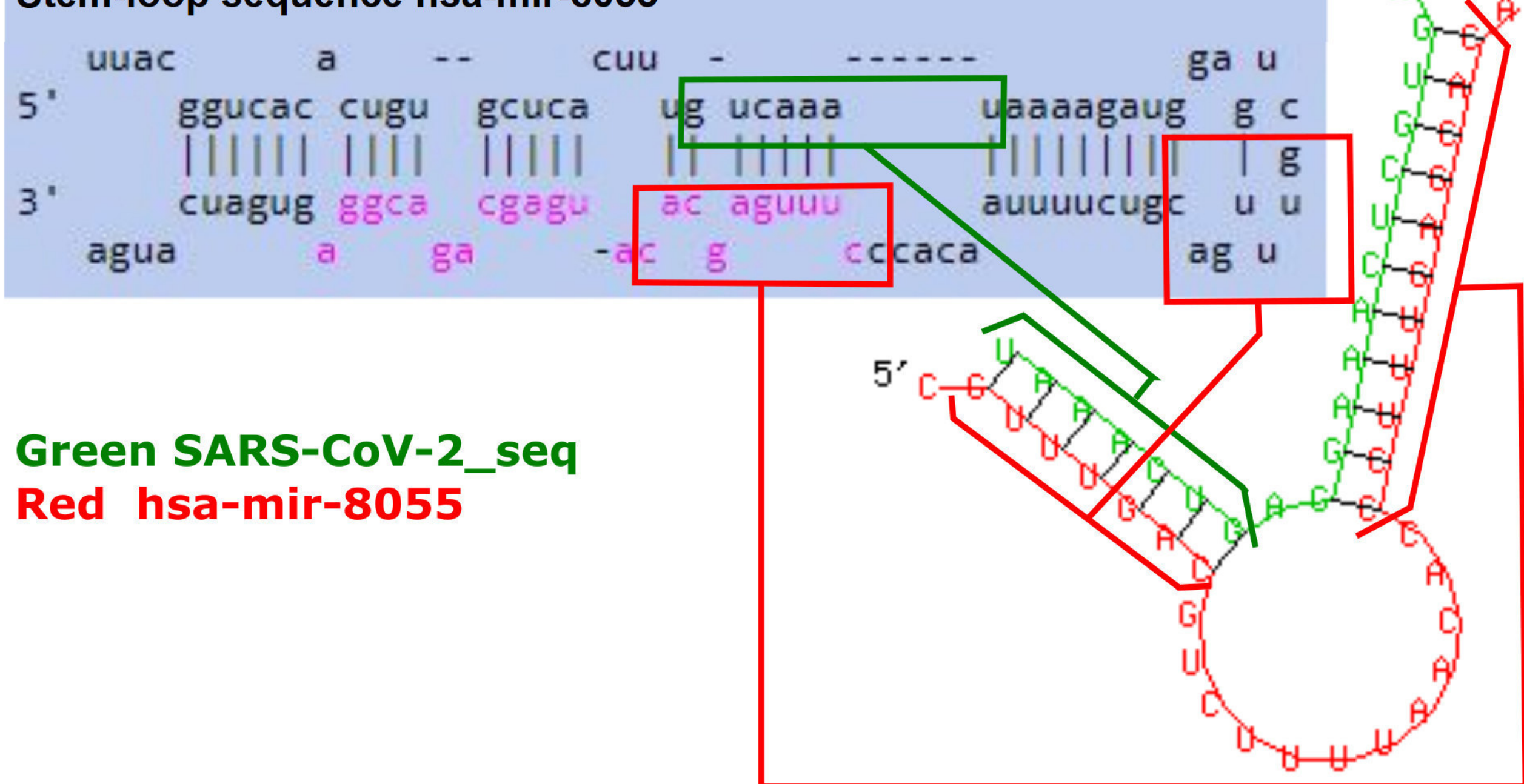

